\title{
MOLECULAR CLOUDS AT HIGH Z
}

\author{
LEO BLITZ \\ Astronomy Program \\ University of Maryland \\ College Park, Maryland, U.S.A. 20742
}

\begin{abstract}
The evidence for the existence of molecular clouds at large distances from the Galactic plane is reviewed. The molecular clouds at high Galactic latitudes are shown to be largely confined to the Galactic plane. There is evidence for one giant molecular cloud as much as four scale heights from the Galactic plane, but given the sample size from which the cloud is drawn, it is reasonable to suppose that it is part of the tail of the thin disk population. There is weak evidence that one star-forming molecular cloud may have originated in the Galactic halo. On the basis of kinematic evidence however, it is shown that there are three molecular clouds identified at high galactic latitude that, if not at high $\mathrm{z}$, are likely to have resulted from interaction with gas in the halo. Understanding how these clouds have formed is likely to be an important key to understanding how the halo interacts with the disk gas.
\end{abstract}

\section{INTRODUCTION}

The existence of molecular gas at large distances from the Galactic plane is of considerable interest because of the unique information the gas would provide about the physical conditions and processes that take place in the halo. As a first step, let us define "large distances" quantitatively. About 100 clouds have been identified at high galactic latitude (see Magnani, Blitz and Mundy 1985, for the initial listing of 57 objects) and several hundred of the appoximately 4000 giant molecular clouds (GMCs) in the Milky Way have been catalogued (e.g. Scoville et al. 1987). It is therefore expected statistically that a few catalogued clouds might be seen at distances as large as $3 \sigma_{h}$ from the Galactic plane, where $\sigma_{h}$ is the rms scale height of the gas. Furthermore, one GMC at $4 \sigma_{h}$ is not highly improbable. We therefore require that a molecular cloud be at least $3 \sigma_{h}$ from the plane to qualify as high-z gas. Because the scale height of the atomic gas is about twice that of the molecular gas (e.g. Knapp 1987, Wouterloot et al. 1990), even gas at four or five $\sigma_{h}$ from the 
plane may not be in contact with the halo gas. Nevertheless, it will be shown below that nearly all of the molecular clouds so far identified appear to be at distances less than $3 \sigma_{h}$ from the Galactic midplane, and only one is known to be as much as $4 \sigma_{h}$.

High $\mathrm{z}$ molecular gas from the ensemble of high latitude clouds can also be identified kinematically. Because the distribution of radial velocities is roughly gaussian (Magnani, Blitz and Mundy 1985, hereafter MBM), clouds that have velocities in excess of $3 \sigma_{v}$, where $\sigma_{v}$ is the one dimensional velocity dispersion of the ensemble, are potentially at high $\mathrm{z}$. On the other hand, the high velocity gas may be locally accelerated in the plane, so detection of such gas requires additional information to place it with certainty at high z. In any event, three clouds have been detected in $\mathrm{CO}$ with velocites from 4 to $8 \sigma_{v}$, which suggests that they might have had some interaction with the halo gas. These clouds are discussed below.

\section{SCALE HEIGHT OF THE MOLECULAR LAYER}

The scale height of the molecular gas is determined primarily by three methods. 1) The angular scale height of $\mathrm{CO}$ is measured at the tangent points of inner Galaxy surveys. The Galactic rotation curve is then used to convert the measurements to a linear scale height. 2) The velocity dispersion of the $\mathrm{CO}$ in the solar vicinity and the midplane density of matter determine the local scale height of $\mathrm{CO}$ if the gas is in equilibrium with the gravitational potential of the disk. 3) A direct measurement of the scale height can be made from HII region/CO complexes with known distances. The first method can be extrapolated to the solar vicinity for comparison with the second. The third method is the only method that can be used in the outer Galaxy. The distances can, in principle, be either spectrophotometric or kinematic, but the latter introduces large uncertainties near the Sun and in regions of known gas streaming such as the Perseus Arm.

The table below gives a number of independently determined values of the local $\mathrm{CO}$ scale height extrapolated from inner Galaxy CO surveys.

TABLE 1. Molecular (CO) Scale Height at $R_{0}$

$\begin{array}{ll}\text { Reference } & \sigma_{\boldsymbol{h}}(\mathrm{pc}) \\ \text { Clemens } \text { et al. }(1988) & 68(85) \\ \text { Dame } \text { et al. }(1987) & 64 \\ \text { Knapp (1987) } & 48 \\ \text { Fich and Blitz (1984) } & 88(101) \\ \text { Wouterloot et al. }(1990) & \sim 85\end{array}$

The first three values are from inner Galaxy CO surveys in which the angular scale height of the $\mathrm{CO}$ is measured at the tangent point along the line of sight (the location of the largest radial velocity) and is then extrapolated to the solar 
distance. The value quoted for Clemens et al.is the mean of values near the solar circle; the parenthetical value is the measured value at $R_{0}$. The Fich and Blitz value is the scale height of the HII region/molecular cloud complexes within $500 \mathrm{pc}$ of $\dot{\mathrm{R}}_{0}$. The quoted value is determined from those objects for which optical distances are available; the parenthetical value is the one quoted in the publication and includes kinematic distances. The Wouterloot et al. value is from their survey of molecular clouds associated with IRAS selected embedded stars, and is extrapolated to $R_{0}$ from measurements at larger Galactic radii.

Taking a straight average of the five determinations gives a gaussian scale height of $71 \pm 16 \mathrm{pc}$. The local scale height of the molecular gas layer may then be taken to be about $70 \mathrm{pc}$ with an uncertainty of about $25 \%$. Molecular clouds at high $\mathrm{z}$ in the solar vicinity are therefore required to be at distances greater than about 200 $\mathrm{pc}$ from the plane.

In the inner Galaxy, the results of all of the $\mathrm{CO}$ surveys show that there is a monotonic increase in the $\mathrm{CO}$ scale height with increasing galactocentric distance. Similarly, in the outer Galaxy, Fich and Blitz (1984) and Wouterlootet al. (1990) also find that the scale height increases with distance. Fich and Blitz find that at $1.4 R_{0}$, the scale height is more than twice the local value, and nearly three times the local value when all objects with distances greater than $1.5 R_{0}$ are averaged together; the Wouterloot et al. observations give reasonable agreement with these values. It is therefore necessary to consider the scale height of the molecular gas at the location in the Galaxy where a particular cloud is located in order to determine whether it is in the plane or not. For example, a molecular cloud located $300 \mathrm{pc}$ from the plane at $R=4 \mathrm{kpc}$ may be reasonably considered to be at the disk-halo interface, whereas a similar object at $\mathrm{R}=15 \mathrm{kpc}$ may not.

\section{ARE THERE MOLECULAR CLOUDS AT HIGH Z?}

\subsection{GMCs in the Solar Vicinity}

A plot of the distances of GMCs from the midplane was given by Blitz (1978), collected from numerous published $\mathrm{CO}$ observations. The more recent all sky $\mathrm{CO}$ survey of Dame et al. (1987) has not appreciably added to the compilation. A number of GMCs are found at distances as much as $150 \mathrm{pc}$ from the Galactic plane including the L1641 cloud in which the Orion Nebula is embedded. None though is farther than $2 \sigma_{h}$ from the plane. Thus, not only are the GMCs in the solar vicinity in the Galactic plane, but all are within one scale height of the atomic gas (Falgarone and Lequeux 1973, Knapp 1987). 


\subsection{High Latitude Molecular Clouds (HLCs)}

The distances to the ensemble of HLCs was initialy determined in two ways (MBM). The first was to assume that they are Galactic plane objects, and assign them a scale height comparable to the other molecular clouds in the plane $(70 \mathrm{pc}$ - see above). By then assuming that they are all at one mean distance from the Sun, the distribution in $b$ will give the mean distance to the ensemble. The second method assumes that the HLCs are in equilibrium with the z-component of the gravitational potential of the disk stars. If the measured radial velocity dispersion of the disk stars is equal to the velocity dispersion in $\mathrm{z}$, then using the Oort limit to the density of matter in the local midplane of the Galaxy (Bahcall 1984) will give the scale height of the HLCs. The angular distribution then gives the mean distance from the Sun. The second method gives a value similar to the one assumed in the first, which provided initial confidence that the mean distance to the HLC ensemble had been reasonably well determined.

Subsequently, a number of direct distance determinations were made to individual HLCs. The first was a measurement of the distances by the classical method of Wolf diagrams (Magnani and deVries 1986). These found the distances to three clouds and upper limits to five more. The distances determined to the eight clouds were consistent with the mean distance of $100 \mathrm{pc}$. Next, in a series of three papers, Hobbs et al. $(1986,1988)$ and Welty et al. $(1989)$ determined the distances to 6 HLCs using the presence or absence of strong $\mathrm{Na} \mathrm{D}$ and $\mathrm{Ca} \mathrm{H}+\mathrm{K}$ absorption toward stars along the line of sight to the HLCs. The stars all have known spectroscopic parallaxes and the distance determinations identified the nearest known molecular cloud (MBM12). Table 2 gives the distances to the clouds for which measurements have been obtained to date.

With the possible exception of MBM 41-44, none of the measured distances imply z-heights that are more than $3 \sigma_{h}$ from the plane. The distance to MBM 41-44 is very uncertain and is discussed separately below. Furthermore, the direct distance measurements imply that the scale height of the HLCs is the same as that determined for the overall population of molecular gas; the measured velocity dispersion of the clouds implies that they are in equilibrium with the gravitational potential of the stars in the disk. Therefore, the molecular clouds in the vicinity of the Sun are found to be Galactic plane objects.

\subsection{Anomalous Velocity Clouds}

Three clouds in the solar vicinity have been found to have velocities well outside the range $3 \sigma_{v}$ for the HLCs, where $\sigma_{v}$ has been measured to be $5.6 \pm 1.2 \mathrm{~km}$ $\mathrm{s}^{-1}(\mathrm{MBM})$. These are G90+38, also known as the Draco Cloud (Georigk et al. 1983) and MBM 41-44 (MBM), with a radial velocity of $-23 \mathrm{~km} \mathrm{~s}^{-1}, \mathrm{G} 211+63$ with a velocity of $-39 \mathrm{~km} \mathrm{~s}^{-1}$ (Désert, Bazell and Blitz 1989), and G135+55 with a velocity of $-45 \mathrm{~km} \mathrm{~s}^{-1}$ (Heiles, Reach and Koo 1988). These clouds have velocities that range from 4-8 $\sigma_{v}$, and are clearly different from the remainder of the HLC 
TABLE 2. Distances to HLCs

$\begin{array}{llll}\text { MBM } & \text { Distance (pc) } & \mathrm{z}(\mathrm{pc}) & \text { (ref.) } \\ 7 & 125 \pm 50 & -75 & 1 \\ 12 & 60-70 & -35 & 2 \\ 16 & 100 \pm 50 & -60 & 1 \\ 16 & 65-95 & \sim-50 & 3 \\ 18 & \leq 175 & \geq-100 & 1 \\ 20 & \leq 125 & \geq-75 & 1 \\ 26 & 175 \pm 50 & 95 & 1 \\ 32 & \leq 275 & \leq 180 & 1 \\ 40 & \leq 140 & \leq 100 & 4 \\ 41-44 & (>800) & (>500) & 5 \\ 53 & 110-155 & \sim-70 & 4 \\ 54 & 145-260 & \sim-120 & 4 \\ 55 & \leq 175 & \leq-110 & 1 \\ 55 & 30 \text { to } 265 & -20 \text { to }-175 & 4 \\ 55 \mathrm{~A} & \leq 275 & \geq-180 & 1\end{array}$

refs:

1 Magnani and deVries (1986)

2 Hobbs, Blitz, and Magnani (1986)

3 Hobbs et al. (1988)

4 Welty et al. (1989)

5 Mebold et al. (1985)

population. If the velocities were representative of motions in equilibrium with the stellar gravitational potential, these clouds would be in the halo of the Galaxy. On the other hand, that all of the velocities are negative suggests that the velocities may represent some other kind of motion, perhaps related to the motions of the classical high-velocity clouds. Whatever their origin, these three clouds are the best candidates for the interaction of molecular gas in the disk-halo connection in the solar vicinity. They will be discussed in more detail below.

\subsection{Galactic Plane GMCs}

Nyman et al. (1987) have called attention to a large molecular cloud in Lupus that appears to be at a much larger distance from the Galactic plane than most of the molecular emission observed in the Dame et al. (1987) CO survey. At a distance of $2.9 \mathrm{kpc}$, the cloud has a distance of $220 \mathrm{pc}$ from the plane. The cloud has a galactocentric distance of $6.7 \mathrm{kpc}$, assuming a value of $8.5 \mathrm{kpc}$ for $\mathrm{R}_{0}$. The $\mathrm{CO}$ scale height at that distance is $\sim 60 \mathrm{pc}$ (using the same surveys discussed in Section 2). The CO cloud in Lupus is therefore about $4 \sigma_{h}$ from the plane. 
Nyman et al. (1987) suggest that because of its large distance from the plane, the Lupus cloud required an extraordinary event in order to form it, and they point to a deficiency in molecular gas at a nearby position in the plane as the site of the postulated explosive event. However, this cloud was identified because it stood out in the survey of inner Galaxy CO. It may therefore be the molecular cloud with the very largest $\mathrm{z}$ height relative to $\sigma_{h}$, and its position might simply be a statistical fluctuation. Recall that for an estimated 4000 GMCs in the Galaxy (Blitz 1978, Solomon and Sanders 1980), one expects $\sim 3$ GMCs in the Galactic ensemble to be at distances that are as large as $4 \sigma_{h}$ from the plane. So, from its position alone, one does not necessarily require an unusual event to form the cloud. Note, however, that the cloud is at a distance of approximately 1.5 atomic scale heights from the plane (Knapp et al. 1987), putting it in the outer portion of the atomic layer but not in what would consider the halo gas. Whatever its origin, the Lupus cloud is unusual, but it nevertheless probably should still be considered a normal Galactic plane object.

\subsection{Young Galactic Clusters}

All of the youngest galactic star clusters are still associated with the gas out of which the stars formed (Bash, Green and Peters, 1977; Leisawitz, Bash and Thaddeus 1989). The latter authors have shown that there are no clusters with ages $<5$ $\times 10^{6} \mathrm{y}$ without associated CO. Furthermore, the clusters that are $<5 \times 10^{6} \mathrm{y}$ old have associated clouds with masses $>10^{4} \mathrm{M}_{\odot}$, and the clusters that are $>10^{7} \mathrm{y}$ old have clouds with masses $<10^{3} \mathrm{M}_{\odot}$ if they have any associated $\mathrm{CO}$ at all. It is therefore reasonable to conclude that clusters with ages of $\sim 10^{7} \mathrm{y}$, are destroying or have recently destroyed the clouds out of which the stars formed.

There are two star clusters, probably associated with one another that provide tantalizing evidence for molecular clouds at high $\mathrm{z}$. Some of the parameters for the two clusters are tabulated below.

\section{TABLE 3. Two Open Clusters at High Z}

$\begin{array}{lll} & \text { NGC } 457 & \text { NGC } 281 \\ l & 1266^{\circ} 56 & 123^{\circ} 13 \\ b & -4.35 & -6.24 \\ \mathrm{~d} & 2-3 \mathrm{kpc} & 2-2.5 \mathrm{kpc} \\ \mathrm{z} & -190 \mathrm{pc} & -240 \mathrm{pc}\end{array}$

NGC 281 contains an 06.5 main sequence star, thus an upper limit to its age is $\sim 3 \times 10^{6} \mathrm{y}$. The cluster also has an associated CO cloud (Leisewitz 1988). NGC 457 , with nearly the same distance and position in the sky, contains an O9.5 star, but no associated $\mathrm{CO}$. The estimated age of the cluster is $10^{7} \mathrm{y}$ from the combined presence of the late $\mathrm{O}$ star and absence of $\mathrm{CO}$. What makes the second cluster so unusual, however, is that it has a measured proper motion of $65 \mathrm{~km} \mathrm{~s}^{-1}$ toward the Galactic plane. The proper motions are thought to be reliable because they 
have been tied to the FK 5 system (de Vecht, personal communication). If the proper motion measurement is correct, it implies that the cluster had an associated molecular cloud that was at one time much farther from the Galactic plane than the cluster is now. If we may take all of the parameters at face value, then $5 \times 10^{6}$ $\mathrm{y}$ ago, the cluster and molecular cloud were $500 \mathrm{pc}$ from the plane! The existence of a molecular cloud associated with NGC 281 lends support to the notion that NGC 457 had a preexisting molecular cloud. However, there are no accurate proper motions for NGC 281.

The tantalizing prospect that a large star forming molecular cloud has formed so far from the Galactic plane rests on the accuracy of the proper motion measurements of the stars in NGC 457 and the correctness of the FK5 system. Confirmation of these proper motions would be good evidence that molecular clouds can exist in the Galactic halo, even though there is no direct evidence among the currently available $\mathrm{CO}$ data sets. Even if the proper motions turn out to be wrong, understanding the reason for the large $\mathrm{z}$ distance of the molecular cloud associated with NGC 281, at more than three scale heights below the plane are as much of a challenge as it is for the Lupus cloud.

\section{THE ANOMALOUS VELOCITY HLCS}

To summarize the previous section, we may conclude that 1) There is no evidence that any local GMCs are not in the thin disk of the Galaxy. 2) There is evidence that an occasional molecular cloud, such as the Lupus cloud, and the NGC 281 cloud are at 3-4 $\sigma_{h}$ of the plane, but there is no strong evidence that these clouds are not at the tail of the thin disk population of molecular clouds. 3) The observational data for the NGC 457 cluster implies that it was formed from a molecular cloud at least $500 \mathrm{pc}$ from the plane. The principal evidence comes from proper motions that need to be confirmed. 4) Direct distance measurements to the HLCs imply that they are part of the thin disk population. 5) There are three HLCs with highly anomalous velocities. Their high negative velocities suggest that they have either fallen from large $\mathrm{z}$ distances, or that they have been accelerated by collisions with infalling gas from the halo.

It appears that the best evidence for a disk-halo interaction among the molecular clouds comes from the anomalous velocity clouds. All three clouds have been the subject of previous studies, and I will summarize the observational data for each of these clouds.

\section{G90+38; The Draco Cloud; MBM 41-44}

Georigk et al. (1983) first called attention to this object because they noticed a high latitude HI feature corresponding to the position of a faint high latitude nebulosity. Furthermore, star counts indicated that there was more extinction than could be accounted by the dust associated with $\mathrm{HI}$; $\mathrm{CO}$ was then sought and 
detected. Subsequently, Mebold et al. (1985), and MBM independently observed $\mathrm{CO}$ associated with the object. MBM called attention to the kinematic anomaly of the CO clouds, and Mebold et al. have attempted to establish a distance to the cloud with the specific aim of trying to ascertain whether the Draco cloud is a molecular cloud in the halo. Using UBV photometry and estimates of the extinction to the clouds, Georigk and Mebold (1986) argued that the distance to the clouds is $>800 \mathrm{pc}$ implying that $\mathrm{z}$ is $>500 \mathrm{pc}$. If correct, it would place this molecular cloud unequivocally at the disk-halo interface.

The existing evidence is, however, weak. On the basis of two lines of sight for the $\mathrm{CO}$, as well as the $\mathrm{HI}$ observations, Mebold et al. argue that the extinction $\left(\mathrm{A}_{v}\right)$ to the region is $\geqslant 2$ mag. The star counts imply much lower extinctions and Mebold et al. concluded that the cloud is more distant than the stars used for the counts. However, the HI data of Georigk et al. (1983) indicate that the mean extinction due to HI is only 0.3 mag. Data given in MBM suggest that the mean extinction due to $\mathrm{H}_{2}$ is no greater than $0.7 \mathrm{mag}$. The mean extinction over the region in which the star counts were made is probably no greater than 0.5 because of the limited extent of the $\mathrm{CO}$ emission. Since the distance of $>800 \mathrm{pc}$ was derived by comparing the star count data to $\mathrm{A}_{v}=2 \mathrm{mag}$, it is probably true that the true distance is less than that derived by Mebold $e t$ al.

Subsequently, Georigk and Mebold (1986) did photometry to 56 stars projected on the Draco atomic hydrogen cloud, and found that the reddeneing to the stars is inconsistent with an extinction of $2 \mathrm{mag}$, and concluded that the stars must be objects in the foreground of the Draco cloud. However, inspection of their colorcolor plot and the reddening line indicates that the locations of the stars may not be inconsistent with a mean extinction of $0.5 \mathrm{mag}$, and thus many of the stars, whose distances are determined from spectrophotometry and argued to be in the foreground, may indeed be background objects.

In any event, the question of the distance can be decided by interstellar absorption line studies of stars projected along the line of sight to the cloud, especially in the direction of the molecular emission. [author's note: A comment from the audience at the meeting mentioned that such observations have, in fact, been completed; the results are eagerly awaited]. Regardless of whether this cloud is in the halo or not, the morphology resembles that of the other two anomalous velocity clouds found to date. That is, the clouds have a cometary morphology suggesting that the highest density portion of the clouds is at the leading portion of the feature.

Odenwald and Rickard (1987), and Odenwald (1988) have catalogued highlatitude cometary objects gleaned from the IRAS data base in order to find other objects that resemble G90+38. They find that the data can be eplained by assuming that dense clouds are moving through the ambient gas with different Reynolds numbers. For G90+38, Odenwald argues that the data suggest that the dense portion of the cloud is moving subsonically through an ambient interstellar medium of low Reynolds number, which suggests that the cloud is indeed at the disk-halo interface and is moving through coronal gas. Other objects appear to be moving through ambient gas of higher viscosity producing turbulent wakes suggestive of 
Mach cones. In the case of $\mathrm{G} 90+38$ the $\mathrm{CO}$ has been extensively mapped, the strongest lines are near the ends of elephant trunks (Mebold, et al. 1985; Rohlfs, et al. 1989) that point in the general direction of the Galactic plane. Odenwald's work suggests that much can be learned about the ambient gas and the dynamics of the disk-halo interface if one can obtain good estimates of the densities in the ambient gas of the general class of cometary structures seen at high Galactic latitudes.

\section{$6135+55$}

This object has the highest radial velocity, $-45 \mathrm{~km} \mathrm{~s}^{-1}$, of any molecular cloud detected at high galactic latitude and was discovered by Heiles, Reach and Koo (1988). The Heiles, Reach and Koo study sought to identify the properties of a sample of 26 isolated clouds at high galactic latitude from the IRAS survey. In their kinematic analysis they concluded that at least two of the clouds in their sample have undergone shocks, and that the shock has modified the grain size distribution. Specifically, they suggest that the very small grains responsible for $12 \mu \mathrm{m}$ emission are destroyed by shocks with velocities of $\sim 10 \mathrm{~km} \mathrm{~s}^{-1}$ and formed in shocks with somewhat higher velocities. Fast shocks, $\gtrsim 40 \mathrm{~km} \mathrm{~s}^{-1}$, are argued to preferentially destroy large grains, thereby elevating the $60 / 100 \mu \mathrm{m}$ flux ratio in clouds. Thus one would expect that the anomalous velocity clouds as a group would exhibit $60 / 100 \mu \mathrm{m}$ flux ratios with values significantly greater than those found in the general interstellar medium. The G135+55 cloud was not otherwise explicitly analyzed in their study.

\section{G211+63}

This cloud has been detected by Désert, Bazell and Blitz (1990), but was independently observed by Heiles, Reach and Koo (personal communication). The cloud was found by Désert et al. in a CO survey of interstellar clouds that exhibit infrared excesses (Désert, Bazell and Boulanger 1988). This cloud is the only one of about 30 new detections of HLCs that has radial velocity more than $2 \sigma_{v}$ (Blitz, Bazell and Désert (1990).

Désert, Bazell and Blitz obtained CO (1-0) and CO (2-1) spectra at the position of infrared emission peak. The 2-1 line observation was made with high frequency resolution and shows a striking double peaked structure. However, no $\mathrm{CO}$ mapping of the source was made. Instead, the authors made carefully constructed maps at $12 \mu \mathrm{m}, 60 \mu \mathrm{m}$, and $100 \mu \mathrm{m}$, and they compared the color ratios of the resulting maps. They conclude that like the cometary globules catalogued by Odenwald (1988), the interaction with the ambient gas is observed as plumes trailing from the back side of the two main dense clumps from which the most intense radiation is detected. The colors suggest that there has been grain processing as the result of shocks that have destroyed the small grains at the leading edge of the object. The brighter clump of dust shows an elevated $60 / 100 \mu \mathrm{m}$ flux ratio as suggested by Heiles, Reach and Koo (1988) for fast shocks. The mass of the dense portion of 
the cloud is estimated to be $1.7 \mathrm{M}_{\odot}$ and the mass of the associated HI (Verschuur $1971)$ is estimated to be $8.7 \mathrm{M}_{\odot}$ at an assumed distance of $100 \mathrm{pc}$. The diameter of the cloud at this distance is $1.2 \mathrm{pc}$.

Based on their velocities, their morphologies, and their infrared properties, the anomalous velocity HLCs seem to be the best candidates to be molecular clouds at the interface between the Galactic disk and the halo. Even if they should turn out not to be at high $\mathrm{z}$, their interaction with the ambient gas can provide useful information about the shock processing of dust and the production of molecules in shocks. On the other hand, if they do turn out to be high $z$ objects, the most important question of all has not been addressed by any of the observations made to date: how did they get there? It is the answer to that question that is most likely to provide the important clues to what is happening at the disk-halo interface.

\section{ACKNOWLEDGEMENTS}

Funding for this work is partially provided by the USNSF grant AST-8918912, and the contribution of the State of Maryland to the Laboratory for Millimeter-wave Astronomy.

\section{REFERENCES}

Bahcall, J.N., 1984, Ap. J., 276, 169.

Bash, F.N., Green, E., and Peters, W.L., 1977, Ap. J., 217, 464.

Blitz, L., 1978, Ph.D. Dissertation, Columbia University.

Blitz, L. Bazell, D, and Désert, F.X., 1990, Ap. J. (Letters), 352, L13.

Clemens, D.P., 1985, Ap. J., 295, 402.

Clemens, D.P., Sanders, D.B., and Scoville, N.Z., 1987, Ap. J., , 327, 139.

Dame, T.M., Elmegreen, B.G., Cohen, R.S., and Thaddeus, P., 1986, Ap. J., 305, 892.

Dame, T.M.. et al., 1987, Ap. J., 322, 706.

Désert, F.X., Bazell, D., and Boulanger, 1988, Ap. J., 334, 815.

Désert, F.X., Bazell, D., and Blitz, L., 1990, Ap. J. (Letters), 355, L51.

Falgarone, E., and Lequeux, J., 1973 Astron. Ap., 25, 253.

Fich, M. and Blitz, L., 1984, Ap. J., 279, 125.

Georigk, W., and Mebold, U., 1986, Astr. Ap, 162, 279.

Georigk, W., Mebold, U., Reif, K., Kalberla, P.M.W., and Velden, L., 1983, Astr. Ap, $120,63$.

Heiles, C., Reach, W.T., and Koo, B.-C., 1988, Ap. J., 322, 313.

Hobbs, L.M., Blitz, L. and Magnani, 1986, Ap. J. (Letters), 306, L109.

Hobbs, L.M., Blitz, L., Penprase, B.E., Magnani, L., and Welty, D.E., 1988, Ap. J., 327, 356.

Knapp. G.R., 1987, Pub. A.S.P., 99, 1134.

Leisawitz, D., 1988, NASA Reference Publication 1202, Catalog of Open Clusters and Associated Interstellar Matter.

Leisawitz, D., Bash, F.N., and Thaddeus, P., 1989, Ap. J. Suppl., 70, 731. 
Magnani, L., Blitz, L., and Mundy, L., 1985, Ap. J., 295, 402.

Magnani, L. and de Vries, C.P., 1986, Astr. Ap, 168, 271.

Mebold, U., Cernicharo, J., Velden, L., Reif, K., Crezelius, C., and Georigk, W., 1985, Astr. Ap, , 151, 427.

Nyman, L.- $\AA$., Thaddeus, P., Bronfman, R.S., and Cohen, R.S., 1987, Ap. J., 314, 374. Odenwald. S.F., 1988, Ap. J., 325, 320.

Odenwald, S.F., and Rickard, L. J, 1987, Ap. J., , 318, 703.

Rohlfs, R., Herbstmeier, U., Mebold, U., and Winnberg, A., 1989, Astr. Ap, 211, 402.

Scoville, N.Z., Yun, M.S., Clemens, D.P., Sanders, D.B., and Waller, W.H., 1987, Ap. J. Suppl., 63, 821 .

Solomon, P.M., and Sanders, D.B., 1980, in Giant Molecular Clouds in the Galaxy, P.M. Solomon, and M.G. Edmunds, eds., Pergamon: Oxford, p.41.

Verschuur, G.L., 1971, Astron. J., 76, 317.

Welty, D.E., Hobbs, L.M., Blitz, L., and Penprase, B.E., 1989, Ap. J., 346, 232.

Wouterloot, J.G.A., Brand, J., Burton, W.B., Kwee, K.K., 1990, Astr. Ap, 230, 21. 


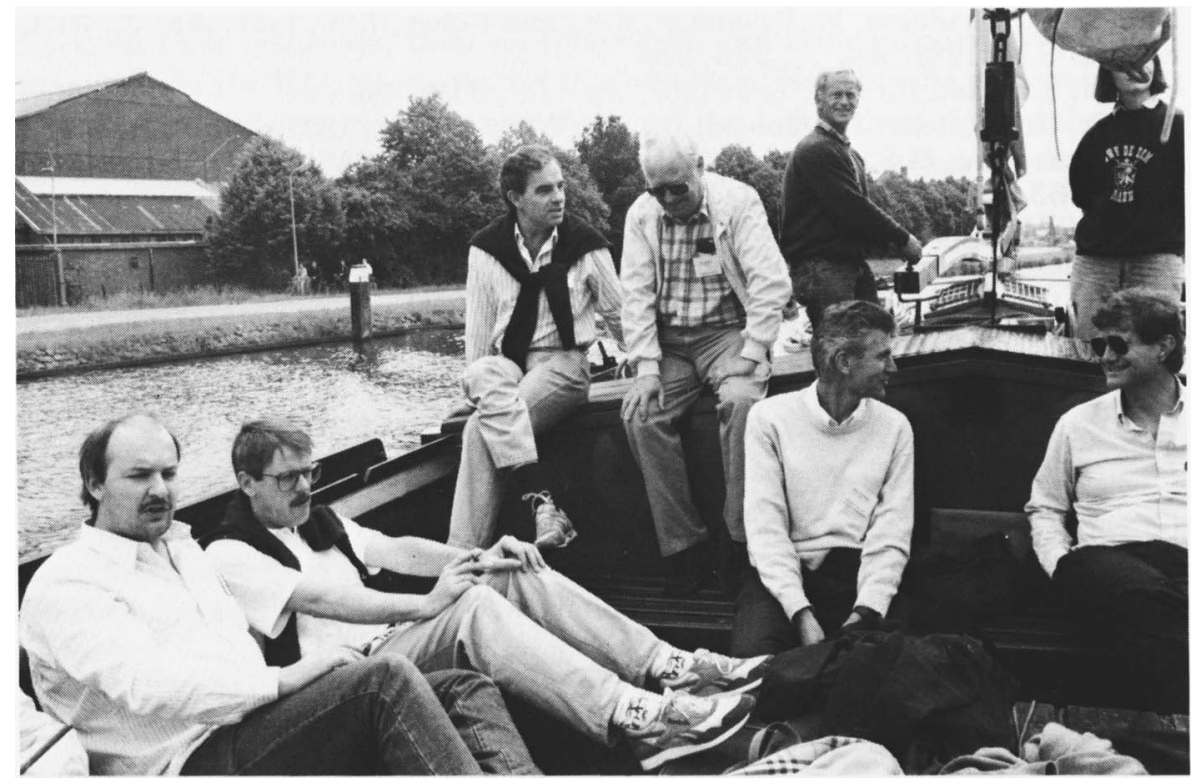

